Article

\title{
Exact Soliton Solutions for Nonlinear Perturbed Schrödinger Equations with Nonlinear Optical Media
}

\author{
Khaled A. Gepreel 1,2 \\ 1 Mathematics Department, Faculty of Science, Taif University, P.O. Box 11099, Taif 21944, Saudi Arabia; \\ kagepreel@yahoo.com or k.gepreel@tu.edu.sa \\ 2 Mathematics Department, Faculty of Sciences, Zagazig University, Zagazig 44519, Egypt
}

Received: 26 November 2020; Accepted: 12 December 2020; Published: 14 December 2020

\begin{abstract}
The nonlinear perturbed Schrödinger equations (NPSEs) with nonlinear terms as Kerr law, power law, quadratic-cubic law, and dual-power law nonlinearity media play an important role in optical fibers. In this article we implement the rational solitary wave method to study the NPSEs when nonlinear terms take some different forms. Additionally, we use the q-deformed hyperbolic function and q-deformed trigonometric function methods to study the exact solutions to NPSEs. Different kind of soliton solutions are obtained such as bright, dark, and singular periodic solutions to the NPSEs.
\end{abstract}

Keywords: nonlinear perturbed Schrödinger equations; Kerr law nonlinearity; nonlinear power law; quadratic-cubic law; anti-cubic law; solitary solutions

\section{Introduction}

In recent years, the research of optical (bright, dark, and singular) solutions for nonlinear Schrödinger equations have played an important role in nonlinear optical media. The optical pulses in the optical solutions play a significant role in communication systems, optical fibers, and so on. Many authors have discussed the optical solutions for the nonlinear Schrödinger equation (NSE) when the nonlinear terms are Kerr law, power law, parabolic law, dual-power law, saturating law, exponential law, higher order polynomial law, and triple-power law (see [1-17]). There are several previous studies which are of great interest in the optical solitons (see [18-22]). Ma et al. [23,24] have discussed exact solutions to NSEs and Lump solutions to higher order rational dispersion relations. Additionally, $\mathrm{Ma}$ [25] has provided better approximations to real physical nonlinear waves, which could exist in even linear wave models. Recently, Arshed [3] studied the exact solution for perturbed Schrödinger equations by using the $\operatorname{Exp}(-\varphi(\xi))$ expansion function method.

In this research, we use the rational solitary solution methods and q-deformed functions methods for the following nonlinear perturbed Schrödinger equation [2-4,7-9]:

$$
i Q_{t}+\sigma Q_{x t}+\varepsilon Q_{x x}+\zeta Q H\left(|Q|^{2}\right)=i\left[\alpha Q_{x}+\eta\left[|Q|^{2 k} Q\right]_{x}+v Q\left(|Q|^{2 k}\right)_{x}\right] .
$$

where $\sigma$ represents the linear evolution phenomena, $\varepsilon$ is dispersion velocity, $\zeta$ is the nonlinear term coefficient, $\alpha$ is the temporal dispersion, $\eta$ is the self-steepening perturbation term, $v$ is the coefficient of nonlinear dispersion, and $k$ is the power of nonlinear terms. We will ansatz the traveling wave transformation as

$$
Q(x, t)=\psi(\xi) e^{i\left(-s_{0} x+w t+\rho\right)}, \xi=x-v t .
$$

where $\psi(\xi)$ is the amplitude real function of the wave solution, $v$ is the soliton velocity, $s_{0}$ is the frequency of the soliton, $w$ is the wave number, and the phase constant is $\rho$. Equation (2) leads to write Equation (1) as 


$$
(\varepsilon-\sigma v) \psi^{\prime \prime}-\left(w+\alpha s_{0}-\sigma w s_{0}+\varepsilon s_{0}^{2}\right) \psi-s_{0} \eta \psi^{2 k+1}+\zeta \psi H\left(\psi^{2}\right)=0
$$

and

$$
\left(v\left(1-\sigma s_{0}\right)-\sigma w+2 \varepsilon s_{0}+\alpha\right)-[(2 k+1) \eta+2 k v] \psi^{2 k}=0 .
$$

Equations (3) and (4) are independent; consequently the soliton velocity is given by

$$
v=\frac{2 \varepsilon s_{0}+\alpha-\sigma w}{\left(\sigma s_{0}-1\right)}, \sigma s_{0}-1 \neq 0
$$

and the constraint condition

$$
(2 k+1) \eta+2 k v=0 .
$$

\section{Analysis of Rational Solitary Solutions Methods (RSSM)}

They are many methods to solve nonlinear partial differential equations (NPDEs) such as transformed rational function (TRF) method [26] and the multiple exp-function (MEF) method [24,25]. The RSSM is one of the important applications to the TRF method and MEF methods for solving the NPDEs. The transformation in Equation (2) is applied to transfer the NPDEs to NODEs. We suppose the solutions of the NODEs according to the RSSM have the shape

$$
W(\xi)=a_{0}+\sum_{i=0}^{N} a_{i} g^{i}(\xi)+\sum_{j=1}^{N} b_{j} g^{j-1}(\xi) f(\xi), \alpha_{N} \neq 0, \text { or } b_{N} \neq 0,
$$

where the conditions on the two functions $g(\xi)$ and $f(\xi)$ take shapes:

$$
\begin{gathered}
f^{\prime}(\xi)=-A g^{2}(\xi)+\frac{\delta A}{B} g(\xi)[1-B g(\xi)], \\
g^{\prime}(\xi)=-A f(\xi) g(\xi), \\
f^{2}(\xi)=g^{2}(\xi)+\frac{\delta}{A^{2}}[1-B g(\xi)]^{2},
\end{gathered}
$$

$A, B, a_{i}, i=0,1,2, \ldots$ and $b_{i}, i=0,1,2, \ldots$ are constants and can be evaluated later, while $\delta= \pm 1$. The nonlinear auxiliary conditions in Equation (8) can be solved as follows:

If $\delta=1$, the hyperbolic solutions take the following form:

$$
f(\xi)=\frac{1}{A \tanh (\xi)+B \operatorname{sech}(\xi)}, \quad g(\xi)=\frac{\operatorname{sech}(\xi)}{A \tanh (\xi)+B \operatorname{sech}(\xi)} .
$$

or

$$
f(\xi)=\frac{\operatorname{coth}(\xi)}{A+B \operatorname{csch}(\xi)}, g(\xi)=\frac{\operatorname{csch}(\xi)}{A+B \operatorname{csch}(\xi)} .
$$

If $\delta=-1$, the rational periodic solutions take the following form:

$$
f(\xi)=\frac{1}{A \tan (\xi)+B \sec (\xi)}, \quad g(\xi)=\frac{\sec (\xi)}{A \tan (\xi)+B \sec (\xi)},
$$

or

$$
f(\xi)=\frac{\cot (\xi)}{A+B \csc (\xi)}, g(\xi)=\frac{\csc (\xi)}{A+B \csc (\xi)} .
$$

The homogenous idea of the balance power is used to determine the end sum of the series in Equation (7). Insert Equation (7) with the auxiliary Equation (8) into the NODEs and deduce the system of algebraic equations from comparison between the coefficients of $f(\xi)$ and $g(\xi)$. Resolve the aforesaid system of equations to $A, B, a_{i}, i=0,1,2, . b_{i}, i=0,1,2, \ldots$ and insert the results in the Equation (3) solution to get the solutions of the NPDEs. 
Remark 1. The system in Equation (8) can be presented by a single of NODE $B g^{\prime \prime}(\xi)=g(\xi)$ $\left[B g^{2}\left\{(\delta+2) A^{2}+\delta B^{2}\right\}-\delta\left(A^{2}+2 B^{2}\right) g+\delta B\right]$ and it has the same solutions (9)-(12) when $A^{2}=B^{2}$.

2.1. Rational Soliton Solution to Nonlinear Perturbed Schrödinger Equations NPSEs with Kerr Law $H(\psi)=\psi$ The nonlinearity Kerr law PNSEs takes the form:

$$
i Q_{t}+\sigma Q_{x t}+\varepsilon Q_{x x}+\zeta Q|Q|^{2}=i\left[\alpha Q_{x}+\eta\left[|Q|^{2 k} Q\right]_{x}+v Q\left(|Q|^{2 k}\right)_{x}\right] .
$$

Equations (3) and (4) lead to write PNSEs in Equation (3) as follows

$$
(\varepsilon-\sigma v) \psi^{\prime \prime}-\left(w+\alpha s_{0}-\sigma w s_{0}+\varepsilon s_{0}^{2}\right) \psi+\left(\zeta-s_{0} \eta\right) \psi^{3}=0 .
$$

when the soliton velocity is given by $v=\frac{2 \varepsilon s_{0}+\alpha-\sigma w}{\left(\sigma s_{0}-1\right)}$, the condition $\eta=\frac{-2 v}{3}$, and put $k=1$ in the nonlinear media to be integrable. Balancing $\psi^{\prime \prime}$ and $\psi^{3}$, we get the Equation (14), which can be written in the form:

$$
\psi(\xi)=a_{0}+a_{1} f(\xi)+b_{1} g(\xi), \alpha_{1} \neq 0, \text { or } b_{1} \neq 0
$$

where $g(\xi), f(\xi)$ satisfies the conditions in Equation $(8)$ and $a_{0}, a_{1}, b_{1}$ are arbitrary constants. The solution formula in Equation (15) is a solution to Equation (14) under the conditions of Equation (8) if and only if:

Family 1. $\delta=1$

$$
\begin{gathered}
a_{0}=0, a_{1}=A \sqrt{\frac{\varepsilon-\sigma v}{2 \eta s_{0}-2 \zeta}},\left(2 \eta s_{0}-2 \zeta\right)(\varepsilon-\sigma v)>0, \eta s_{0}-\zeta \neq 0, \\
b_{1}=\sqrt{\frac{\left(A^{2}+B^{2}\right)(\varepsilon-\sigma v)}{2 \eta s_{0}-2 \zeta}}, w=\frac{2 \alpha s_{0}+2 \varepsilon s_{0}^{2}+\varepsilon-\sigma v}{2\left(\sigma s_{0}-1\right)} .
\end{gathered}
$$

In this family the solution of PNSEs in Equation (13) take the form:

$$
\begin{aligned}
Q_{1}(x, t)= & e^{i\left(-s_{0} x+w t+\rho\right)\left[\sqrt{\frac{\varepsilon-\sigma v}{2 \eta s_{0}-2 \zeta}}\right.} \frac{A}{A \tanh (x-v t)+B \operatorname{sech}(x-v t)} \\
& \left.+\sqrt{\frac{\left(A^{2}+B^{2}\right)(\varepsilon-\sigma v)}{2 \eta s_{0}-2 \zeta}} \frac{\operatorname{sech}(x-v t)}{A \tanh (x-v t)+B \operatorname{sech}(x-v t)}\right]
\end{aligned}
$$

or

$$
\begin{aligned}
Q_{2}(x, t) & =e^{i\left(-s_{0} x+w t+\rho\right)}\left[\sqrt{\frac{\varepsilon-\sigma v}{2 \eta s_{0}-2 \zeta}} \frac{A \operatorname{coth}(x-v t)}{A+B \operatorname{csch}(x-v t)}\right. \\
& \left.+\sqrt{\frac{\left(A^{2}+B^{2}\right)(\varepsilon-\sigma v)}{2 \eta s_{0}-2 \zeta}} \frac{\operatorname{csch}(x-v t)}{A+B \operatorname{csch}(x-v t)}\right]
\end{aligned}
$$

Family 2. $\delta=-1$

$$
\begin{gathered}
a_{0}=0, a_{1}=A \sqrt{\frac{\varepsilon-\sigma v}{2 \eta s_{0}-2 \zeta}},(\varepsilon-\sigma v)\left(2 \eta s_{0}-2 \zeta\right)>0, \eta s_{0}-\zeta \neq 0 \\
b_{1}=\sqrt{\frac{\left(A^{2}-B^{2}\right)(\varepsilon-\sigma v)}{2 \eta s_{0}-2 \zeta}}, A>B, w=\frac{2 \alpha s_{0}+2 \varepsilon s_{0}^{2}-\varepsilon+\sigma v}{2\left(\sigma s_{0}-1\right)} .
\end{gathered}
$$

In this family, the solution of PNSEs in Equation (13) take the form:

$$
\begin{aligned}
& Q_{3}(x, t)=e^{i\left(-s_{0} x+w t+\rho\right)}\left[\sqrt{\frac{\varepsilon-\sigma v}{2 \eta s_{0}-2 \zeta}} \frac{A}{A \tan (x-v t)+B \sec (x-v t)}\right. \\
& \left.+\sqrt{\frac{\left(A^{2}-B^{2}\right)(\varepsilon-\sigma v)}{2 \eta s_{0}-2 \zeta}} \frac{\sec (x-v t)}{A \tan (x-v t)+B \sec (x-v t)}\right],
\end{aligned}
$$

or

$$
\begin{aligned}
Q_{4}(x, t)= & e^{i\left(-s_{0} x+w t+\rho\right)\left[\sqrt{\frac{\varepsilon-\sigma v}{2 \eta s_{0}-2 \zeta}}\right.} \frac{A \cot (x-v t)}{A+B \csc (x-v t)} \\
& \left.+\sqrt{\frac{\left(A^{2}-B^{2}\right)(\varepsilon-\sigma v)}{2 \eta s_{0}-2 \zeta}} \frac{\csc (x-v t)}{A+B \csc (x-v t)}\right] .
\end{aligned}
$$


2.2. Rational Soliton Solution to NPSEs with Power Law $H(\psi)=\psi^{k}, 0<k<0$

The nonlinearity power law PNSEs take the form:

$$
i Q_{t}+\sigma Q_{x t}+\varepsilon Q_{x x}+\zeta Q|Q|^{2 k}=i\left[\alpha Q_{x}+\eta\left[|Q|^{2 k} Q\right]_{x}+v Q\left(|Q|^{2 k}\right)_{x}\right] .
$$

Equations (3) and (4) lead to write PNSEs in Equation (22) as follows:

$$
(\varepsilon-\sigma v) \psi^{\prime \prime}-\left(w+\alpha s_{0}-\sigma w s_{0}+\varepsilon s_{0}^{2}\right) \psi+\left(\zeta-s_{0} \eta\right) \psi^{2 k+1}=0,
$$

when the soliton velocity is given by $v=\frac{2 \varepsilon s_{0}+\alpha-\sigma w}{\left(\sigma s_{0}-1\right)}$ and $\eta=\frac{-2 k v}{2 k+1}$. From balance the highest power of $\psi^{\prime \prime}$ and $\psi^{2 k+1}$, we get $N=\frac{1}{k}$ as rational number, and we take the transformation

$$
\psi(\xi)=[\varphi(\xi)]^{1 / 2 k}
$$

The transformation in Equation (24) leads to obtain

$$
(\varepsilon-\sigma v)(1-2 k) \varphi^{\prime 2}+2 k(\varepsilon-\sigma v) \varphi \varphi^{\prime \prime}-4 k^{2}\left(w+\alpha s_{0}-\sigma w s_{0}+\varepsilon s_{0}^{2}\right) \varphi^{2}+4 k^{2}\left(\zeta-s_{0} \eta\right) \varphi^{3}=0 .
$$

From $\varphi \varphi^{\prime \prime}$ and $\varphi^{3}$, the solution of Equation (21) can be written in the form:

$$
\psi(\xi)=a_{0}+a_{1} f(\xi)+b_{1} g(\xi)+a_{2} f(\xi) g(\xi)+b_{2} g^{2}(\xi), \alpha_{2} \neq 0, \text { or } b_{2} \neq 0,
$$

where $g(\xi), f(\xi)$ satisfies the conditions in Equation (8), and $a_{0}, a_{1}, a_{2}, b_{1}, b_{2}$ are arbitrary constants. The solution formula in Equation (26) is a solution to Equation (25) under conditions (8) if and only if:

Family 3. $\delta=1$

$$
\begin{gathered}
a_{2}=\frac{A(k+1)(v \sigma-\varepsilon) \sqrt{A^{2}+B^{2}}}{2 k^{2}\left(\eta \varepsilon_{0}-\zeta\right)}, a_{1}=a_{0}=0, \eta s_{0}-\zeta \neq 0 \\
b_{1}=\frac{B(k+1)(v \sigma-\varepsilon)}{2 k^{2}\left(\eta s_{0}-\zeta\right)}, w=\frac{v \sigma-\varepsilon+4 k^{2} s_{0} \alpha+4 k^{2} s_{0}^{2} \varepsilon}{4 k^{2}\left(\sigma s_{0}-1\right)}, \\
b_{2}=-\frac{\left(A^{2}+B^{2}\right)(k+1)(v \sigma-\varepsilon)}{2 k^{2}\left(\eta s_{0}-\zeta\right)} .
\end{gathered}
$$

In this family the solution of PNSEs in Equation (22) takes the form:

$$
\begin{gathered}
Q_{5}(x, t)=e^{i\left(-s_{0} x+w t+\rho\right)}\left[\frac{B(k+1)(v \sigma-\varepsilon) \operatorname{sech}(x-v t)}{2 k^{2}\left(\eta s_{0}-\zeta\right)[A \tanh (x-v t)+B \operatorname{sech}(x-v t)]}\right. \\
\left.+\frac{A(k+1)(v \sigma-\varepsilon) \sqrt{A^{2}+B^{2}} \operatorname{sech}(x-v t)}{2 k^{2}\left(\eta s_{0}-\zeta\right)[A \tanh (x-v t)+B \operatorname{sech}(x-v t)]^{2}}-\frac{\left(A^{2}+B^{2}\right)(k+1)(v \sigma-\varepsilon) \operatorname{sech}^{2}(x-v t)}{2 k^{2}\left(\eta s_{0}-\zeta\right)[A \tanh (x-v t)+B \operatorname{sech}(x-v t)]^{2}}\right]^{1 / 2 k}
\end{gathered}
$$

or

$$
\begin{gathered}
Q_{6}(x, t)=e^{i\left(-s_{0} x+w t+\rho\right)}\left[\frac{B(k+1)(v \sigma-\varepsilon) \operatorname{coth}(x-v t)}{2 k^{2}\left(\eta s_{0}-\zeta\right)[A+B \operatorname{csch}(x-v t)]}\right. \\
\left.+\frac{A(k+1)(v \sigma-\varepsilon) \sqrt{A^{2}+B^{2} \operatorname{coth}(x-v t) \operatorname{csch}(x-v t)}}{2 k^{2}\left(\eta s_{0}-\zeta\right)[A+B \operatorname{csch}(x-v t)]^{2}}-\frac{\left(A^{2}+B^{2}\right)(k+1)(v \sigma-\varepsilon) \operatorname{csch}^{2}(x-v t)}{\left.2 k^{2}\left(\eta s_{0}-\zeta\right)[A+B \operatorname{csch}(x-v t))\right]^{2}}\right]^{1 / 2 k}
\end{gathered}
$$

Family 4. $\delta=-1$

$$
\begin{gathered}
a_{2}=\frac{A(k+1)(v \sigma-\varepsilon) \sqrt{A^{2}-B^{2}}}{2 k^{2}\left(\eta s_{0}-\zeta\right)}, a_{1}=a_{0}=0, A^{2}-B^{2}>0, \eta s_{0}-\zeta \neq 0 \\
b_{1}=-\frac{B(k+1)(v \sigma-\varepsilon)}{2 k^{2}\left(\eta s_{0}-\zeta\right)}, w=\frac{-v \sigma+\varepsilon+4 k^{2} s_{0} \alpha+4 k^{2} s_{0}^{2} \varepsilon}{4 k^{2}\left(\sigma s_{0}-1\right)}, \\
b_{2}=-\frac{\left(A^{2}-B^{2}\right)(k+1)(v \sigma-\varepsilon)}{2 k^{2}\left(\eta s_{0}-\zeta\right)} .
\end{gathered}
$$


In this family, the solution of PNSEs in Equation (22) take the form:

$$
\begin{gathered}
Q_{7}(x, t)=e^{i\left(-s_{0} x+w t+\rho\right)}\left[-\frac{B(k+1)(v \sigma-\varepsilon) \sec (x-v t)}{2 k^{2}\left(\eta s_{0}-\zeta\right)[A \tan (x-v t)+B \sec (x-v t)]}\right. \\
\left.+\frac{A(k+1)(v \sigma-\varepsilon) \sqrt{A^{2}-B^{2}} \sec (x-v t)}{2 k^{2}\left(\eta s_{0}-\zeta\right)[A \tan (x-v t)+B \sec (x-v t)]^{2}}-\frac{\left(A^{2}-B^{2}\right)(k+1)(v \sigma-\varepsilon) \sec ^{2}(x-v t)}{2 k^{2}\left(\eta s_{0}-\zeta\right)[A \tan (x-v t)+B \sec (x-v t)]^{2}}\right]^{1 / 2 k} .
\end{gathered}
$$

or

$$
\begin{gathered}
Q_{8}(x, t)=e^{i\left(-s_{0} x+w t+\rho\right)}\left[-\frac{B(k+1)(v \sigma-\varepsilon) \csc (x-v t)}{2 k^{2}\left(\eta s_{0}-\zeta\right)[A+B \csc (x-v t)]}\right. \\
\left.+\frac{A(k+1)(v \sigma-\varepsilon) \sqrt{A^{2}-B^{2}} \cot (x-v t) \csc (x-v t)}{2 k^{2}\left(\eta s_{0}-\zeta\right)[A+B \csc (x-v t)]^{2}}-\frac{\left(A^{2}-B^{2}\right)(k+1)(v \sigma-\varepsilon) \csc ^{2}(x-v t)}{2 k^{2}\left(\eta s_{0}-\zeta\right)[A+B \csc (x-v t)]^{2}}\right]^{1 / 2 k} .
\end{gathered}
$$

There are many different other solutions, but we leave it as a kind of convenience.

\subsection{Rational Soliton Solution to NPSEs with Quadratic-Cubic Law $H(Q)=E_{1} \sqrt{Q}+E_{2} Q$}

The nonlinearity quadratic law PNSEs take the form:

$$
i Q_{t}+\sigma Q_{x t}+\varepsilon Q_{x x}+\zeta\left[E_{1}|Q|+E_{2}|Q|^{2}\right] Q=i\left[\alpha Q_{x}+\eta\left[|Q|^{2 k} Q\right]_{x}+v Q\left(|Q|^{2 k}\right)_{x}\right]
$$

where $E_{1}$ and $E_{2}$ are constants. Equations (3) and (4) lead to write PNSEs in Equation (33) as follows:

$$
(\varepsilon-\sigma v) \psi^{\prime \prime}-\left(w+\alpha s_{0}-\sigma w s_{0}+\varepsilon s_{0}^{2}\right) \psi+E_{1} \psi^{2}+E_{2} \psi^{3}-s_{0} \eta \psi^{2 k+1}=0 .
$$

When the soliton velocity is given by $v=\frac{2 \varepsilon s_{0}+\alpha-\sigma w}{\left(\sigma s_{0}-1\right)}$, the condition $\eta=\frac{-2 k v}{2 k+1}$. Balancing the power of $\psi^{\prime \prime}$ and $\psi^{3}$ in the series solution in Equation (7) with the restriction in Equation (8), we have $N=1$ and Equation (28) is integrable when $k=1$. Consequently, we set the solution in the following form:

$$
\psi(\xi)=a_{0}+a_{1} f(\xi)+b_{1} g(\xi) \alpha_{1} \neq 0, \text { or } b_{1} \neq 0,
$$

where $g(\xi), f(\xi)$ satisfies the conditions in Equation (8), and $a_{0}, a_{1}, b_{1}$ are arbitrary constants. The solution formula in Equation (35) is a solution to Equation (34) under conditions in Equation (8) if and only if:

Family 5. $\delta=1$

$$
\begin{gathered}
a_{0}=\frac{-E_{1}}{3\left(E_{2}-s_{0} \eta\right)}, a_{1}=\frac{A E_{1}}{3\left(E_{2}-s_{0} \eta\right)}, \\
b_{1}=\frac{E_{1} \sqrt{A^{2}+B^{2}}}{3\left(E_{2}-s_{0} \eta\right)}, \sigma=\frac{2 E_{1}^{2}+9 E_{2} \varepsilon-9 s_{0} \varepsilon \eta}{9 v\left(E_{2}-s_{0} \eta\right)}, E_{2}-s_{0} \eta \neq 0 \\
w=\frac{v\left(-9 \varepsilon s_{0}^{3} \eta+9 \varepsilon s_{0}^{2} E_{2}-9 \alpha s_{0}^{2} \eta+9 \alpha s_{0} E_{2}+2 E_{1}^{2}\right)}{9 s_{0} \varepsilon E_{2}+9 v s_{0} \eta-9 s_{0}^{2} \varepsilon \eta-9 v E_{2}+2 s_{0} E_{1}^{2}} .
\end{gathered}
$$

In this family the solution of PNSEs in Equation (33) take the form:

$$
\begin{gathered}
Q_{9}(x, t)=e^{i\left(-s_{0} x+w t+\rho\right)}\left[\frac{-E_{1}}{3\left(E_{2}-s_{0} \eta\right)}+\frac{A E_{1}}{3\left(E_{2}-s_{0} \eta\right)[A \tanh (x-v t)+B \operatorname{sech}(x-v t)]}\right. \\
\left.+\frac{E_{1} \sqrt{A^{2}+B^{2}} \operatorname{sech}(x-v t)}{3\left(E_{2}-s_{0} \eta\right)[A \tanh (x-v t)+B \operatorname{sech}(x-v t)]}\right]
\end{gathered}
$$

or

$$
\begin{gathered}
Q_{10}(x, t)=e^{i\left(-s_{0} x+w t+\rho\right)}\left[\frac{-E_{1}}{3\left(E_{2}-s_{0} \eta\right)}+\frac{A E_{1} \operatorname{coth}(x-v t)}{3\left(E_{2}-s_{0} \eta\right)[A+B \operatorname{csch}(x-v t)]}\right. \\
\left.+\frac{E_{1} \sqrt{A^{2}+B^{2}} \operatorname{csch}(x-v t)}{3\left(E_{2}-s_{0} \eta\right)[A+B \operatorname{csch}(x-v t)]}\right] .
\end{gathered}
$$


Family 6. $\delta=-1$

$$
\begin{gathered}
b_{1}=-\frac{3 B a_{0}\left(2 E_{2} a_{0}-2 s_{0} \eta a_{0}+E_{1}\right)}{3 E_{2} a_{0}+E_{1}-3 s_{0} \eta a_{0}}, A=\frac{B \sqrt{2 E_{1}^{2}-3 s_{0} E_{1} \eta a_{0}+3 E_{1} E_{2} a_{0}}}{\sqrt{2}\left[3 E_{2} a_{0}+E_{1}-3 s_{0} \eta a_{0}\right]}, \\
w=\frac{v\left(-s_{0} \eta a_{0}^{2}+E_{1} a_{0}+E_{2} a_{0}^{2}-\varepsilon s_{0}^{2}-\alpha s_{0}\right)}{2 s_{0} E_{2} a_{0}^{2}-2 s_{0}^{2} \eta a_{0}^{2}-s_{0} \varepsilon+v+s_{0} E_{1} a_{0}}, \sigma=-\frac{1}{v}\left(2 E_{2} a_{0}^{2}-2 s_{0} \eta a_{0}^{2}+E_{1} a_{0}-\varepsilon\right), \\
a_{1}=0.2 E_{1}^{2}-3 s_{0} E_{1} \eta a_{0}+3 E_{1} E_{2} a_{0}>0
\end{gathered}
$$

In this family, the solution of PNSEs in Equation (33) takes the form:

$$
Q_{11}=\left[a_{0}-\frac{3 a_{0}\left(2 E_{2} a_{0}-2 s_{0} \eta a_{0}+E_{1}\right) \sec (x-v t)}{\left[\sqrt{2 E_{1}^{2}-3 s_{0} E_{1} \eta a_{0}+3 E_{1} E_{2} a_{0}} \tan (x-v t)+\left(3 E_{2} a_{0}+E_{1}-3 s_{0} \eta a_{0}\right) \sec (x-v t)\right]}\right] e^{i\left(-s_{0} x+w t+\rho\right)} .
$$

or

$$
Q_{12}(x, t)=\left[a_{0}-\frac{3 a_{0}\left(2 E_{2} a_{0}-2 s_{0} \eta a_{0}+E_{1}\right) \csc (x-v t)}{\left[\sqrt{2 E_{1}^{2}-3 s_{0} E_{1} \eta a_{0}+3 E_{1} E_{2} a_{0}}+\left(3 E_{2} a_{0}+E_{1}-3 s_{0} \eta a_{0}\right) \csc (x-v t)\right]}\right] e^{i\left(-s_{0} x+w t+\rho\right)} .
$$

There are many different other families, but we leave it as a kind of convenience to the readers.

2.4. Rational Soliton Solution to NPS's with Anti-Cubic Law $H(Q)=\frac{M_{1}}{Q^{2}}+M_{2} Q+M_{3} Q^{2}$

The nonlinearity anti cubic law PNSEs takes the form:

$$
i Q_{t}+\sigma Q_{x t}+\varepsilon Q_{x x}+\zeta\left[M_{1}|Q|^{-4}+M_{2}|Q|^{2}+M_{3}|Q|^{4}\right] Q=i\left[\alpha Q_{x}+\eta\left[|Q|^{2 k} Q\right]_{x}+v Q\left(|Q|^{2 k}\right)_{x}\right],
$$

where $M_{1}, M_{2}$ and $M_{3}$ are nonzero constants. Equations (3) and (4) lead to write PNSEs in Equation (34) as follows:

$$
(\varepsilon-\sigma v) \psi^{3} \psi^{\prime \prime}-\left(w+\alpha s_{0}-\sigma w s_{0}+\varepsilon s_{0}^{2}\right) \psi^{4}-4 s_{0} \eta \psi^{2 k+4}+M_{1}+M_{2} \psi^{6}+M_{3} \psi^{8}=0 .
$$

when the soliton velocity is given by $v=\frac{2 \varepsilon s_{0}+\alpha-\sigma w}{\left(\sigma s_{0}-1\right)}$, the condition $\eta=\frac{-2 k v}{2 k+1}$. From equating the power of terms $\psi^{3} \psi^{\prime \prime}$ and We suppose $N=1 / 2$, we have $\psi^{8}$

$$
\psi=[\Omega(\xi)]^{1 / 2} .
$$

From Equations (44) and (43) we get:

$$
(\varepsilon-\sigma v)\left[2 \Omega \Omega^{\prime \prime}-\Omega^{\prime 2}\right]-4\left(w+\alpha s_{0}-\sigma w s_{0}+\varepsilon s_{0}^{2}\right) \Omega^{2}-4 s_{0} \eta \Omega^{4}+4\left(M_{1}+M_{2} \Omega^{3}+M_{3} \Omega^{4}\right)=0 .
$$

Consequently, we set the solution of Equation (45) in the following form:

$$
\Omega(\xi)=a_{0}+a_{1} f(\xi)+b_{1} g(\xi) \alpha_{1} \neq 0, \text { or } b_{1} \neq 0,
$$

where $g(\xi), f(\xi)$ satisfies the conditions in Equation (8), and $a_{0}, a_{1}, b_{1}$ are arbitrary constants. The solution formula in Equation (46) is a solution to Equation (45) under conditions of Equation (8) if and only if: 
Family 7. $\delta=1$

$$
\begin{gathered}
M_{1}=-\frac{a_{0}^{2}(\sigma v-\varepsilon)}{4 b_{1}^{2}}\left[2 a_{0} b_{1} B+a_{0}^{2}\left(A^{2}+B^{2}\right)+b_{1}^{2}\right], a_{1}=0, \\
M_{2}=-\frac{(\sigma v-\varepsilon)}{b_{1}^{2}}\left[2 a_{0}\left(A^{2}+B^{2}\right)+B b_{1}\right], b_{1} \neq 0 \\
M_{3}=\frac{1}{4 b_{1}^{2}}\left[3(\sigma v-\varepsilon)\left(A^{2}+B^{2}\right)+4 s_{0} \eta b_{1}^{2}\right], \\
w=\frac{1}{4 b_{1}^{2}\left(\sigma s_{0}-1\right)}\left[6 a_{0}^{2}(\sigma v-\varepsilon)\left(A^{2}+B^{2}\right)+(\sigma v-\varepsilon)\left(b_{1}^{2}+6 a_{0} b_{1} B\right)+4 \varepsilon s_{0}^{2} b_{1}^{2}+4 \alpha s_{0} b_{1}^{2}\right] .
\end{gathered}
$$

In this family, the solution of PNSEs in Equation (42) takes the form:

$$
Q_{13}(x, t)=e^{i\left(-s_{0} x+w t+\rho\right)}\left[a_{0}+\frac{b_{1} \operatorname{sech}(x-v t)}{[A \tanh (x-v t)+B \operatorname{sech}(x-v t)]}\right]^{1 / 2} .
$$

or

$$
Q_{14}(x, t)=e^{i\left(-s_{0} x+w t+\rho\right)}\left[a_{0}+\frac{b_{1} \operatorname{csch}(x-v t)}{[A+B \operatorname{csch}(x-v t)]}\right]^{1 / 2} .
$$

Family 8. $\delta=-1$

$$
\begin{gathered}
M_{1}=\frac{a_{0}^{2}(\sigma v-\varepsilon)}{4 b_{1}^{2}}\left[2 a_{0} b_{1} B-a_{0}^{2}\left(A^{2}-B^{2}\right)+b_{1}^{2}\right], a_{1}=0, \\
M_{2}=\frac{(\sigma v-\varepsilon)}{b_{1}^{2}}\left[-2 a_{0}\left(A^{2}-B^{2}\right)+B b_{1}\right], \\
M_{3}=\frac{1}{4 b_{1}^{2}}\left[3(\sigma v-\varepsilon)\left(A^{2}-B^{2}\right)+4 s_{0} \eta b_{1}^{2}\right], \\
w=\frac{1}{4 b_{1}^{2}\left(\sigma s_{0}-1\right)}\left[6 a_{0}^{2}(\sigma v-\varepsilon)\left(A^{2}-B^{2}\right)-(\sigma v-\varepsilon)\left(b_{1}^{2}+6 a_{0} b_{1} B\right)+4 \varepsilon s_{0}^{2} b_{1}^{2}+4 \alpha s_{0} b_{1}^{2}\right] .
\end{gathered}
$$

In this family, the solution of PNSEs in Equation (42) takes the form:

$$
Q_{15}(x, t)=e^{i\left(-s_{0} x+w t+\rho\right)}\left[a_{0}+\frac{b_{1} \sec (x-v t)}{[A \tan (x-v t)+B \sec (x-v t)]}\right]^{1 / 2},
$$

or

$$
Q_{16}(x, t)=e^{i\left(-s_{0} x+w t+\rho\right)}\left[a_{0}+\frac{b_{1} \csc (x-v t)}{[A+B \csc (x-v t)]}\right]^{1 / 2}
$$

There are many different other families, but we leave it as a kind convenience to the readers.

\section{Q-Deformed Hyperbolic Functions Method to NPSEs}

Many authors have introduced the definition of q-deformed hyperbolic functions (see, for example, $[7,12-14])$. The q-deformed hyperbolic functions are defined by $\sinh _{q}(\xi)=\frac{1}{2}\left(e^{\xi}-q e^{-\xi}\right)$, and $\cosh _{q}(\xi)=\frac{1}{2}\left(e^{\xi}+q e^{-\xi}\right)$. There are many applications of q-deformed hyperbolic and trigonometric functions to NPDEs (see [26]). In the q-deformed hyperbolic functions methods, we suppose the solution of NODEs in the following form:

Type 1.

$$
\psi(\xi)=A_{0}+\sum_{i=1}^{n}\left(\operatorname{sech}_{q} \xi\right)^{i-1}\left(A_{i} \operatorname{sech}_{q} \xi+B_{i} \tanh _{q} \xi\right)
$$

where

$$
\left(\operatorname{sech}_{q} \xi\right)^{\prime}=-\operatorname{sech}_{q} \xi \tanh _{q} \xi,\left(\tanh _{q} \xi\right)^{\prime}=q \operatorname{sech}_{q}^{2}(\xi), q \operatorname{sech}_{q}^{2}(\xi)+\tanh _{q}^{2} \xi=1
$$

Type 2.

$$
\varphi(\xi)=A_{0}+\sum_{i=1}^{n}\left(\operatorname{csch}_{q} \xi\right)^{i-1}\left(A_{i} \operatorname{csch}_{q} \xi+B_{i} \operatorname{coth}_{q} \xi\right)
$$


where

$$
\left(\operatorname{csch}_{q} \xi\right)^{\prime}=-\operatorname{csch}_{q} \xi \operatorname{coth}_{q} \xi,\left(\operatorname{coth}_{q} \xi\right)^{\prime}=q \operatorname{csch}_{q}^{2}(\xi), q \operatorname{csch}_{q}^{2}(\xi)+\operatorname{coth}_{q}^{2} \xi=1 .
$$

\section{1. $\operatorname{Kerr} \operatorname{Law} H(\psi)=\psi$}

From Type 1, we suppose the solution to NPSEs in Equation (14) in the form in Equation (53). The formula in Equation (53) is a solution to Equation (14) when $n=1$, hence

$$
\psi(\xi)=A_{0}+A_{1} \operatorname{sech}_{q} \xi+B_{1} \tanh _{q} \xi
$$

Substituting Equation (57) into Equation (14) and setting the coefficients of $\left(\operatorname{sech}_{q} \xi\right)^{j}\left(\tanh _{q} \xi\right)^{i}$, $j=0,1, i=0,1,2, \ldots$, to be zero, we acquire the system equations, which can be solved to get:

Set 1.

$$
A_{0}=0, A_{1}=\sqrt{\frac{2 q(\sigma v-\varepsilon)}{\eta s_{0}-\varsigma}}, B_{1}=0, w=\frac{\varepsilon s_{0}^{2}+\alpha s_{0}+\sigma v-\varepsilon}{\sigma s_{0}-1}, q>0,(\sigma v-\varepsilon)\left(\eta s_{0}-\zeta\right)>0 .
$$

In this set, the solution to NPSEs in Equation (13) is

$$
Q_{17}(x, t)=\sqrt{\frac{2 q(\sigma v-\varepsilon)}{\eta s_{0}-\varsigma}} e^{i\left(-s_{0} x+w t+\rho\right)} \operatorname{sech}_{q}(x-v t)
$$

Set 2.

$$
A_{0}=0, A_{1}=\sqrt{\frac{q(\sigma v-\varepsilon)}{2\left(\eta s_{0}-\varsigma\right)}}, B_{1}=\sqrt{\frac{-(\sigma v-\varepsilon)}{2\left(\eta s_{0}-\varsigma\right)}}, w=\frac{\sigma v-2 \varepsilon s_{0}^{2}-2 \alpha s_{0}-\varepsilon}{2\left(1-\sigma s_{0}\right)}, q<0, \frac{(\sigma v-\varepsilon)}{\eta s_{0}-\varsigma}<0 .
$$

In this set, the solution to NPSEs in Equation (13) is

$$
Q_{18}(x, t)=e^{i\left(-s_{0} x+w t+\rho\right)}\left[\sqrt{\frac{q(\sigma v-\varepsilon)}{2\left(\eta s_{0}-\varsigma\right)}} \operatorname{sech}_{q}(x-v t)+\sqrt{\frac{-(\sigma v-\varepsilon)}{2\left(\eta s_{0}-\varsigma\right)}} \tanh _{q}(x-v t)\right]
$$

For the type 2. the solutions of the NPSEs in Equation (13) are given by:

$$
\begin{gathered}
Q_{19}(x, t)=e^{i\left(-s_{0} x+w t+\rho\right)}\left[\sqrt{\frac{2 q(\varepsilon-\sigma v)}{\eta s_{0}-\varsigma}} \operatorname{csch}_{q}(x-v t)\right], \frac{q(\varepsilon-\sigma v)}{v s_{0}-\eta}>0, w=\frac{\varepsilon s_{0}^{2}+\sigma v-\varepsilon+\alpha s_{0}}{\sigma s_{0}-1} \\
Q_{20}(x, t)=e^{i\left(-s_{0} x+w t+\rho\right)}\left[\sqrt{\frac{2(\varepsilon-\sigma v)}{\eta s_{0}-\varsigma}} \operatorname{coth}_{q}(x-v t)\right], \frac{(\varepsilon-\sigma v)}{\eta s_{0}-\varsigma}>0, w=\frac{\varepsilon s_{0}^{2}+\alpha s_{0}+2 \varepsilon-2 \sigma v}{\sigma s_{0}-1}, \\
Q_{21}(x, t)=e^{i\left(-s_{0} x+w t+\rho\right)}\left[\sqrt{\frac{q(\varepsilon-\sigma v)}{2\left(\eta s_{0}-\varsigma\right)}} \operatorname{csch}_{q}(x-v t)+\sqrt{\frac{\varepsilon-\sigma v}{2\left(\eta s_{0}-\zeta\right)}} \operatorname{coth}_{q}(x-v t)\right] \\
\frac{(\varepsilon-\sigma v)}{\eta s_{0}-\varsigma}>0, w=\frac{2 \varepsilon s_{0}^{2}+2 \alpha s_{0}+\varepsilon-\sigma v}{2 \sigma s_{0}-1}
\end{gathered}
$$

\subsection{Power $\operatorname{Law} H(\psi)=\psi^{k}, 0<k<2$}

From Type 1, we suppose the solution to NPSEs in Equation (25) in the form in Equation (53). The formula in Equation (53) is a solution to Equation (25) when $n=2$, consequently we have:

$$
\psi(\xi)=A_{0}+A_{1} \operatorname{sech}_{q} \xi+B_{1} \tanh _{q} \xi+A_{2} \operatorname{sech}_{q}^{2} \xi+B_{2} \operatorname{sech}_{q} \xi \tanh _{q} \xi .
$$

Equation (65) is a solution to Equation (25) when 
Set 1.

$$
\begin{gathered}
A_{2}=\frac{q(k+1)(\sigma v-\varepsilon)}{2 k^{2}\left(\eta s_{0}-\varsigma\right)}, B_{2}=\frac{\sqrt{-q}(k+1)(\sigma v-\varepsilon)}{2 k^{2}\left(\eta s_{0}-\varsigma\right)}, w=\frac{4 \varepsilon s_{0}^{2} k^{2}+4 \alpha s_{0} k^{2}+\sigma v-\varepsilon}{4 k^{2}\left(\sigma s_{0}-1\right)}, q<0, \\
A_{0}=A_{1}=B_{1}=0 .
\end{gathered}
$$

In this set, the solution to NPSEs in Equation (22) is

$$
Q_{22}(x, t)=e^{i\left(-s_{0} x+w t+\rho\right)}\left[\frac{q(k+1)(\sigma v-\varepsilon)}{2 k^{2}\left(\eta s_{0}-\varsigma\right)} \operatorname{sech}^{2}{ }_{q} \xi+\frac{\sqrt{-q}(k+1)(\sigma v-\varepsilon)}{2 k^{2}\left(\eta s_{0}-\varsigma\right)} \operatorname{sech}_{q} \xi \tanh _{q} \xi\right]^{1 / 2 k}
$$

Set 2.

$$
A_{2}=\frac{q(k+1)(\sigma v-\varepsilon)}{2 k^{2}\left(\eta s_{0}-\varsigma\right)}, w=\frac{\varepsilon s_{0}^{2} k^{2}+\alpha s_{0} k^{2}+\sigma v-\varepsilon}{k^{2}\left(\sigma s_{0}-1\right)}, A_{0}=A_{1}=B_{1}=B_{2}=0,
$$

In this set, the solution to NPSEs in Equation (22) is

$$
Q_{23}(x, t)=e^{i\left(-s_{0} x+w t+\rho\right)}\left[\frac{q(k+1)(\sigma v-\varepsilon)}{2 k^{2}\left(\eta s_{0}-\varsigma\right)} \operatorname{sech}_{q}^{2} \xi\right]^{1 / 2 k}
$$

For the type 2. The solutions of the NPSEs in Equation (22) are given by:

$$
Q_{22}(x, t)=e^{i\left(-s_{0} x+w t+\rho\right)}\left[-\frac{q(k+1)(\sigma v-\varepsilon)}{2 k^{2}\left(\eta s_{0}-\varsigma\right)} \operatorname{csch}_{q}^{2}(\xi)+\frac{\sqrt{q}(k+1)(\sigma v-\varepsilon)}{2 k^{2}\left(\eta s_{0}-\varsigma\right)} \operatorname{csch}_{q} \xi \operatorname{coth}_{q} \xi\right]^{1 / 2 k}
$$

where $w=\frac{4 \varepsilon s_{0}^{2} k^{2}+4 \alpha s_{0} k^{2}+\sigma v-\varepsilon}{4 k^{2}\left(\sigma s_{0}-1\right)}$ and $q>0$

$$
Q_{23}(x, t)=e^{i\left(-s_{0} x+w t+\rho\right)}\left[\frac{-q(k+1)(\sigma v-\varepsilon)}{k^{2}\left(\eta s_{0}-\varsigma\right)} \operatorname{csch}_{q}^{2} \xi\right]^{1 / 2 k} .
$$

where $w=\frac{\varepsilon s_{0}^{2} k^{2}+\alpha s_{0} k^{2}+\sigma v-\varepsilon}{k^{2}\left(\sigma s_{0}-1\right)}$.

\subsection{Quadratic-Cubic Law $H(Q)=E_{1} \sqrt{Q}+E_{2} Q$}

From Type 1, we suppose the solution to NPSEs in Equation (34) in the form in Equation (53). The formula in Equation (53) is a solution to Equation (34) when $n=1$, and Equation (34) is integrable when $k=1$. Suppose a solution of Equation (34) in the following form:

$$
\psi(\xi)=A_{0}+A_{1} \operatorname{sech}_{q} \xi+B_{1} \tanh _{q} \xi .
$$

Equation (72) is a solution of Equation (34) when

Set 1.

$A_{0}=\frac{-6(\sigma v-\varepsilon)}{E_{1}}, A_{1}=0, B_{1}=\frac{6(\sigma v-\varepsilon)}{E_{1}}, w=\frac{\varepsilon s_{0}^{2}+\alpha s_{0}+4 \sigma v-4 \varepsilon}{\sigma s_{0}-1}, E_{2}=\frac{18 s_{0} \eta(\sigma v-\varepsilon)+E_{1}^{2}}{18(\sigma v-\varepsilon)}$.

In this set, the solution to NPSEs in Equation (33) is

$$
Q_{24}(x, t)=\left[\frac{-6(\sigma v-\varepsilon)}{E_{1}}+\frac{6(\sigma v-\varepsilon)}{E_{1}} \tanh _{q}(x-v t)\right] e^{i\left(-s_{0} x+w t+\rho\right)} .
$$

Set 2.

$$
A_{0}=\frac{3(\sigma v-\varepsilon)}{E_{1}}, A_{1}=\frac{3 \sqrt{2 q}(\sigma v-\varepsilon)}{E_{1}}, B_{1}=0, w=\frac{\varepsilon s_{0}^{2}+\alpha s_{0}-2 \sigma v+2 \varepsilon}{\sigma s_{0}-1}, E_{2}=-\frac{-9 s_{0} \eta(\sigma v-\varepsilon)+E_{1}^{2}}{9(\sigma v-\varepsilon)}, q>0 .
$$


In this set, the solution to NPSEs in Equation (33) is

$$
Q_{25}(x, t)=\left[\frac{3(\sigma v-\varepsilon)}{E_{1}}+\frac{3 \sqrt{2 q}(\sigma v-\varepsilon)}{E_{1}} \operatorname{sech}_{q}(x-v t)\right] e^{i\left(-s_{0} x+w t+\rho\right)} .
$$

Set 3.

$$
\begin{aligned}
A_{0} & =\frac{-3(\sigma v-\varepsilon)}{2 E_{1}}, A_{1}=\frac{3(\sigma v-\varepsilon) \sqrt{-q}}{2 E_{1}}, B_{1}=\frac{3(\sigma v-\varepsilon)}{2 E_{1}}, \\
w & =\frac{\varepsilon s_{0}^{2}+\alpha s_{0}+\sigma v-\varepsilon}{\sigma s_{0}-1}, E_{2}=\frac{9 s_{0} \eta(\sigma v-\varepsilon)+2 E_{1}^{2}}{9(\sigma v-\varepsilon)}, q<0
\end{aligned}
$$

In this set, the solution to NPSEs in Equation (33) is

$$
Q_{26}(x, t)=\left[\frac{-3(\sigma v-\varepsilon)}{2 E_{1}}+\frac{3(\sigma v-\varepsilon) \sqrt{-q}}{2 E_{1}} \operatorname{sech}_{q}(x-v t)+\frac{3(\sigma v-\varepsilon)}{2 E_{1}} \tanh _{q}(x-v t)\right] e^{i\left(-s_{0} x+w t+\rho\right)} .
$$

For the type 2. The solutions of the NPSEs in Equation (33) are given by

$$
Q_{27}(x, t)=\left[-\frac{6(\sigma v-\varepsilon)}{E_{1}}-\frac{6(\sigma v-\varepsilon)}{E_{1}} \operatorname{coth}_{q}(x-v t)\right] e^{i\left(s_{0} x+w t+\rho\right)},
$$

where $w=\frac{\varepsilon s_{0}^{2}+\alpha s_{0}+4(\sigma v-\varepsilon)}{\left(\sigma s_{0}-1\right)}$ and $E_{2}=\frac{18 s_{0} \eta(\sigma v-\varepsilon)+E_{1}^{2}}{18(\sigma v-\varepsilon)}$.

$$
Q_{28}(x, t)=\left[\frac{3(\sigma v-\varepsilon)}{E_{1}}+\frac{3 \sqrt{-2 q}(\sigma v-\varepsilon)}{E_{1}} \operatorname{csch}_{q}(x-v t)\right] e^{i\left(s_{0} x+w t+\rho\right)} .
$$

where $w=\frac{\varepsilon s_{0}^{2}+\alpha s_{0}-2 \sigma v+2 \varepsilon}{\sigma s_{0}-1}$ and $E_{2}=-\frac{-9 s_{0} \eta(\sigma v-\varepsilon)+E_{1}^{2}}{9(\sigma v-\varepsilon)}, q<0$.

$$
Q_{29}(x, t)=\left[\frac{-3(\sigma v-\varepsilon)}{2 E_{1}}+\frac{3(\sigma v-\varepsilon) \sqrt{q}}{2 E_{1}} \operatorname{csch}_{q}(x-v t)-\frac{3(\sigma v-\varepsilon)}{2 E_{1}} \operatorname{coth}_{q}(x-v t)\right] e^{i\left(-s_{0} x+w t+\rho\right)} .
$$

where $w=\frac{\varepsilon s_{0}^{2}+\alpha s_{0}+(\sigma v-\varepsilon)}{\left(\sigma s_{0}-1\right)}$ and $E_{2}=\frac{9 s_{0} \eta(\sigma v-\varepsilon)+2 E_{1}^{2}}{9(\sigma v-\varepsilon)}, q>0$.

3.4. Anti-Cubic Law $H(Q)=M_{1} Q^{-2}+M_{2} Q+M_{3} Q^{2}$

From Type 1, we suppose the solution to NPSEs in Equation (45) in the form in Equation (53). The formula in Equation (53) is a solution to Equation (45) when $n=1$. Suppose a solution of Equation (45) in the following form:

$$
\Omega(\xi)=A_{0}+A_{1} \operatorname{sech}_{q} \xi+B_{1} \tanh _{q} \xi,
$$

Equation (82) is a solution of Equation (45) when

Set 1.

$$
\begin{gathered}
A_{1}=0, B_{1}=\sqrt{\frac{3(\sigma v-\varepsilon)}{4\left(M_{3}-\eta s_{0}\right)}}, \frac{(\sigma v-\varepsilon)}{\left(M_{3}-\eta s_{0}\right)}>0, w=\frac{4 A_{0}^{2}\left(M_{3}-\eta s_{0}\right)+2 \varepsilon s_{0}^{2}+2 \alpha s_{0}+\varepsilon-\sigma v}{2\left(\sigma s_{0}-1\right)}, \\
M_{2}=-\frac{8 A_{0}}{3}\left(M_{3}-\eta s_{0}\right), M_{1}=-\frac{1}{48\left(M_{3}-\eta s_{0}\right)}\left[\left(4 M_{3} A_{0}^{2}-3 \sigma v\right)+\left(3 \varepsilon-4 \eta s_{0} A_{0}^{2}\right)\right]^{2} .
\end{gathered}
$$

In this set, the solution to NPSEs in Equation (45) is

$$
Q_{30}(x, t)=\left[A_{0}+\sqrt{\frac{3(\sigma v-\varepsilon)}{4\left(M_{3}-\eta s_{0}\right)}} \tanh _{q}(x-v t)\right]^{1 / 2} e^{i\left(-s_{0} x+w t+\rho\right)} .
$$


Set 2.

$$
\begin{gathered}
B_{1}=0, A_{1}=\sqrt{\frac{3 q(\sigma v-\varepsilon)}{4\left(M_{3}-\eta s_{0}\right)}}, \frac{q(\sigma v-\varepsilon)}{\left(M_{3}-\eta s_{0}\right)}>0, w=\frac{8 A_{0}^{2}\left(M_{3}-\eta s_{0}\right)+4 \varepsilon s_{0}^{2}+4 \alpha s_{0}+\sigma v-\varepsilon}{4\left(\sigma s_{0}-1\right)}, \\
M_{2}=-\frac{8}{3} A_{0}\left(M_{3}-\eta s_{0}\right), M_{1}=-\frac{A_{0}^{2}}{12}\left[4 A_{0}^{2}\left(M_{3}-\eta s_{0}\right)+3(\sigma v-\varepsilon)\right] .
\end{gathered}
$$

In this set, the solution to NPSEs in Equation (42) is

$$
Q_{31}(x, t)=\left[A_{0}+\sqrt{\frac{3 q(\sigma v-\varepsilon)}{4\left(M_{3}-\eta s_{0}\right)}} \operatorname{sech}_{q}(x-v t)\right]^{1 / 2} e^{i\left(-s_{0} x+w t+\rho\right)} .
$$

Set 3 .

$$
\begin{gathered}
A_{1}=\frac{1}{4} \sqrt{\frac{-3 q(\sigma v-\varepsilon)}{\left(M_{3}-\eta s_{0}\right)}}, B_{1}=\frac{1}{4} \sqrt{\frac{3(\sigma v-\varepsilon)}{\left(M_{3}-\eta s_{0}\right)}}, \frac{(\sigma v-\varepsilon)}{\left(M_{3}-\eta s_{0}\right)}>0, q<0, M_{2}=-\frac{8 A_{0}}{3}\left(M_{3}-\eta s_{0}\right), \\
w=\frac{16 A_{0}^{2}\left(M_{3}-\eta s_{0}\right)+8 \varepsilon s_{0}^{2}+8 \alpha s_{0}+\varepsilon-\sigma v}{8\left(\sigma s_{0}-1\right)}, M_{1}=-\frac{1}{768\left(M_{3}-\eta s_{0}\right)}\left[\left(16 M_{3} A_{0}^{2}+3 \varepsilon\right)-\left(16 \eta s_{0} A_{0}^{2}+3 \sigma v\right)\right]^{2} .
\end{gathered}
$$

In this set, the solution to NPSEs in Equation (42) is

$$
Q_{32}(x, t)=\left[A_{0}+\frac{1}{4} \sqrt{\frac{-3 q(\sigma v-\varepsilon)}{\left(M_{3}-\eta s_{0}\right)}} \operatorname{sech}_{q}(x-v t)+\frac{1}{4} \sqrt{\frac{3(\sigma v-\varepsilon)}{\left(M_{3}-\eta s_{0}\right)}} \tanh _{q}(x-v t)\right]^{1 / 2} e^{i\left(-s_{0} x+w t+\rho\right)} .
$$

For the type 2. The solutions of the NPSEs in Equation (42) are given by

$$
Q_{33}(x, t)=\left[A_{0}+\sqrt{\frac{3(\sigma v-\varepsilon)}{4\left(M_{3}-\eta s_{0}\right)}} \operatorname{coth}_{q}(x-v t)\right]^{1 / 2} e^{i\left(-s_{0} x+w t+\rho\right)}, \frac{3(\sigma v-\varepsilon)}{4\left(M_{3}-\eta s_{0}\right)}>0,
$$

where $M_{1}=-\frac{1}{48\left(M_{3}-\eta s_{0}\right)}\left[\left(4 M_{3} A_{0}^{2}-3 \sigma v\right)+\left(3 \varepsilon-4 \eta s_{0} A_{0}^{2}\right)\right]^{2}, M_{2}=-\frac{8}{3}\left(M_{3}-\eta s_{0}\right)$, and $w=$ $\frac{4 A_{0}^{2}\left(M_{3}-\eta s_{0}\right)+2 \varepsilon s_{0}^{2}+2 \alpha s_{0}+\varepsilon-\sigma v}{2\left(\sigma s_{0}-1\right)}$.

$$
Q_{34}(x, t)=\left[A_{0}+\sqrt{\frac{3 q(\sigma v-\varepsilon)}{4\left(M_{3}-\eta s_{0}\right)}} \operatorname{csch}_{q}(x-v t)\right]^{1 / 2} e^{i\left(-s_{0} x+w t+\rho\right)}, \frac{3 q(\sigma v-\varepsilon)}{4\left(M_{3}-\eta s_{0}\right)}>0,
$$

where $M_{1}=-\frac{A_{0}^{2}}{12}\left[4 A_{0}^{2}\left(M_{3}-\eta s_{0}\right)+3(\sigma v-\varepsilon)\right], \quad M_{2}=-\frac{8}{3} A_{0}\left(M_{3}-\eta s_{0}\right), \quad$ and $w=$ $\frac{8 A_{0}^{2}\left(M_{3}-\eta s_{0}\right)+4 \varepsilon s_{0}^{2}+4 \alpha s_{0}+\sigma v-\varepsilon}{4\left(\sigma s_{0}-1\right)}$.

$$
Q_{35}(x, t)=\left[A_{0}+\frac{1}{4} \sqrt{\frac{3 q(\sigma v-\varepsilon)}{\left(M_{3}-\eta s_{0}\right)}} \operatorname{csch}_{q}(x-v t)+\frac{1}{4} \sqrt{\frac{3(\sigma v-\varepsilon)}{\left(M_{3}-\eta s_{0}\right)}} \operatorname{coth}_{q}(x-v t)\right]^{1 / 2} e^{i\left(-s_{0} x+w t+\rho\right)} .
$$

where $\frac{3(\sigma v-\varepsilon)}{\left(M_{3}-\eta s_{0}\right)}>0, M_{1}=-\frac{1}{768\left(M_{3}-\eta s_{0}\right)}\left[\left(16 M_{3} A_{0}^{2}+3 \varepsilon\right)-\left(16 \eta s_{0} A_{0}^{2}+3 \sigma v\right)\right]^{2}$ and $q>0, M_{2}=$ $-\frac{8 A_{0}}{3}\left(M_{3}-\eta s_{0}\right)$ and $w=\frac{16 A_{0}^{2}\left(M_{3}-\eta s_{0}\right)+8 \varepsilon s_{0}^{2}+8 \alpha s_{0}+\varepsilon-\sigma v}{8\left(\sigma s_{0}-1\right)}$.

\section{Q-Deformed Trigonometric Functions Method}

Many authors have defined the q-deformed trigonometric functions in $[7,12-14,26]$ as $\sin _{q}(\xi)=$ $\frac{1}{2 i}\left(e^{i \xi}-q e^{-i \xi}\right)$, and $\cos _{q}(\xi)=\frac{1}{2}\left(e^{i \xi}+q e^{-i \xi}\right)$. In the q-deformed trigonometric functions methods, we suppose the solution of Equation (14) in the following form: 
Type 3.

$$
\psi(\xi)=A_{0}+\sum_{i=0}^{n}\left(\sec _{q} \xi\right)^{i-1}\left(A_{i} \sec _{q} \xi+B_{i} \tan _{q} \xi\right)
$$

where

$$
\left(\sec _{q} \xi\right)^{\prime}=\sec _{q} \xi \tan _{q} \xi, \quad\left(\tan _{q} \xi\right)^{\prime}=q \sec _{q}^{2}(\xi),{q \sec _{q}}^{2}(\xi)-\tan _{q}^{2} \xi=1
$$

Type 4.

$$
\psi(\xi)=A_{0}+\sum_{i=0}^{n}\left(\csc _{q} \xi\right)^{i-1}\left(A_{i} \csc _{q} \xi+B_{i} \cot _{q} \xi\right)
$$

where

$$
\left(\csc _{q} \xi\right)^{\prime}=-\csc _{q} \xi \cot _{q} \xi, \quad\left(\cot _{q} \xi\right)^{\prime}=-q \csc _{q}^{2}(\xi), \quad q \csc _{q}^{2}(\xi)-\cot _{q}^{2} \xi=1
$$

4.1. $\operatorname{Kerr} \operatorname{Law} H(\psi)=\psi$

From Type 3, we suppose the solution to NPSEs in Equation (14) in the form in Equation (92). The formula in Equation (92) is a solution to Equation (14) when $n=1$, hence

$$
\psi(\xi)=A_{0}+A_{1} \sec _{q} \xi+B_{1} \tan _{q} \xi .
$$

Substituting Equation (96) into Equation (14) and setting the coefficients of $\left(\sec _{q} \xi\right)^{j}\left(\tan _{q} \xi\right)^{i}$, $\mathrm{j}=0,1, \mathrm{i}=0,1,2, \ldots$, to be zero, we acquire the system equations which can be solved to have:

Set 1.

$$
A_{0}=0, A_{1}=\sqrt{\frac{2 q(\varepsilon-\sigma v)}{\eta s_{0}-\varsigma}}, \quad B_{1}=0, w=\frac{\varepsilon s_{0}^{2}+\alpha s_{0}-\sigma v+\varepsilon}{\sigma s_{0}-1}, q>0, \frac{\varepsilon-\sigma v}{\eta s_{0}-\zeta}>0 .
$$

In this set, the solution to NPSEs in Equation (13) is

$$
Q_{36}(x, t)=\sqrt{\frac{2 q(\varepsilon-\sigma v)}{\eta s_{0}-\varsigma}} e^{i\left(-s_{0} x+w t+\rho\right)} \sec _{q}(x-v t)
$$

Set 2 .

$$
A_{0}=0, A_{1}=0, \quad B_{1}=\sqrt{\frac{2(\varepsilon-\sigma v)}{\eta s_{0}-\varsigma}}, w=\frac{\varepsilon s_{0}^{2}+\alpha s_{0}+2 \sigma v-2 \varepsilon}{\sigma s_{0}-1}, \frac{\varepsilon-\sigma v}{\eta s_{0}-\zeta}>0 .
$$

In this set, the solution to NPSEs in Equation (13) is

$$
Q_{37}(x, t)=\sqrt{\frac{2(\varepsilon-\sigma v)}{\eta s_{0}-\varsigma}} e^{i\left(-s_{0} x+w t+\rho\right)} \tan _{q}(x-v t)
$$

Set 3.

$A_{0}=0, A_{1}=\sqrt{\frac{q(\varepsilon-\sigma v)}{2\left(\eta s_{0}-\varsigma\right)}}, B_{1}=\sqrt{\frac{(\varepsilon-\sigma v)}{2\left(\eta s_{0}-\varsigma\right)}}, w=\frac{\sigma v+2 \varepsilon s_{0}^{2}+2 \alpha s_{0}-\varepsilon}{2\left(\sigma s_{0}-1\right)}, q>0, \frac{(\varepsilon-\sigma v)}{\left(\eta s_{0}-\varsigma\right)}>0$. 
In this set, the solution to NPSEs in Equation (13) is

$$
Q_{38}(x, t)=e^{i\left(-s_{0} x+w t+\rho\right)}\left[\sqrt{\frac{q(\varepsilon-\sigma v)}{2\left(\eta s_{0}-\varsigma\right)}} \sec _{q}(x-v t)+\sqrt{\frac{(\varepsilon-\sigma v)}{2\left(\eta s_{0}-\varsigma\right)}} \tan _{q}(x-v t)\right]
$$

For the type 4. The solutions of the NPSEs in Equation (13) are given by:

$$
Q_{39}(x, t)=\sqrt{\frac{2 q(\varepsilon-\sigma v)}{\eta s_{0}-\varsigma}} e^{i\left(-s_{0} x+w t+\rho\right)} \csc _{q}(x-v t)
$$

where $w=\frac{\varepsilon s_{0}^{2}+\alpha s_{0}-\sigma v+\varepsilon}{\sigma s_{0}-1}, q>0$, and $\frac{\varepsilon-\sigma v}{\eta s_{0}-\zeta}>0$.

$$
Q_{40}(x, t)=\sqrt{\frac{2(\varepsilon-\sigma v)}{\eta s_{0}-\varsigma}} e^{i\left(-s_{0} x+w t+\rho\right)} \cot _{q}(x-v t)
$$

where $w=\frac{\varepsilon s_{0}^{2}+\alpha s_{0}+2 \sigma v-2 \varepsilon}{\sigma s_{0}-1}, q>0$, and $\frac{\varepsilon-\sigma v}{\eta s_{0}-\zeta}>0$.

$$
Q_{41}(x, t)=e^{i\left(-s_{0} x+w t+\rho\right)}\left[\sqrt{\frac{q(\varepsilon-\sigma v)}{2\left(\eta s_{0}-\varsigma\right)}} \csc _{q}(x-v t)+\sqrt{\frac{(\varepsilon-\sigma v)}{2\left(\eta s_{0}-\varsigma\right)}} \cot _{q}(x-v t)\right],
$$

where $w=\frac{\sigma v+2 \varepsilon s_{0}^{2}+2 \alpha s_{0}-\varepsilon}{2\left(\sigma s_{0}-1\right)}$ and $\frac{q(\varepsilon-\sigma v)}{\left(\eta s_{0}-\varsigma\right)}>0$.

4.2. Power $\operatorname{Law} H(\psi)=\psi^{k}, 0<k<2$

From Type 3, we suppose the solution to NPSEs in Equation (25) in the form in Equation (92). The formula in Equation (92) is a solution to Equation (25) when $n=2$; consequently we have:

$$
\psi(\xi)=A_{0}+A_{1} \sec _{q} \xi+B_{1} \tan _{q} \xi+A_{2} \sec ^{2}{ }_{q} \xi+B_{2} \sec _{q} \xi \tan _{q} \xi
$$

Equation (106) is a solution to Equation (25) when

\section{Set 1.}

$$
\begin{gathered}
A_{2}=-\frac{q(k+1)(\sigma v-\varepsilon)}{2 k^{2}\left(\eta s_{0}-\varsigma\right)}, \quad B_{2}=\frac{\sqrt{q}(k+1)(\sigma v-\varepsilon)}{2 k^{2}\left(\eta s_{0}-\varsigma\right)}, w=\frac{4 \varepsilon s_{0}^{2} k^{2}+4 \alpha s_{0} k^{2}-\sigma v+\varepsilon}{4 k^{2}\left(\sigma s_{0}-1\right)}, q>0, \\
A_{0}=A_{1}=B_{1}=0,
\end{gathered}
$$

In this set, the solution to NPSEs in Equation (22) is

$$
Q_{42}(x, t)=e^{i\left(-s_{0} x+w t+\rho\right)}\left[-\frac{q(k+1)(\sigma v-\varepsilon)}{2 k^{2}\left(\eta s_{0}-\varsigma\right)} \sec ^{2} q(x-v t)+\frac{\sqrt{q}(k+1)(\sigma v-\varepsilon)}{2 k^{2}\left(\eta s_{0}-\varsigma\right)} \sec _{q}(x-v t) \tan _{q}(x-v t)\right]^{1 / 2 k} .
$$

Set 2 .

$$
A_{2}=\frac{q(k+1)(\sigma v-\varepsilon)}{2 k^{2}\left(\eta s_{0}-\varsigma\right)}, w=\frac{\varepsilon s_{0}^{2} k^{2}+\alpha s_{0} k^{2}-\sigma v+\varepsilon}{k^{2}\left(\sigma s_{0}-1\right)}, A_{0}=A_{1}=B_{1}=B_{2}=0,
$$

In this set, the solution to NPSEs in Equation (22) is

$$
Q_{43}(x, t)=e^{i\left(-s_{0} x+w t+\rho\right)}\left[\frac{q(k+1)(\sigma v-\varepsilon)}{2 k^{2}\left(\eta s_{0}-\varsigma\right)} \sec _{q}^{2}(x-v t)\right]^{\frac{1}{2 k}} .
$$


For the type 4. The solutions of the NPSEs in Equation (22) are given by:

$$
Q_{43}(x, t)=e^{i\left(-s_{0} x+w t+\rho\right)}\left[-\frac{q(k+1)(\sigma v-\varepsilon)}{2 k^{2}\left(\eta s_{0}-\varsigma\right)} \csc ^{2} q(x-v t)+\frac{\sqrt{q}(k+1)(\sigma v-\varepsilon)}{2 k^{2}\left(\eta s_{0}-\varsigma\right)} \csc _{q}(x-v t) \cot _{q}(x-v t)\right]^{\frac{1}{2 k}} .
$$

where $w=\frac{4 \varepsilon s_{0}^{2} k^{2}+4 \alpha s_{0} k^{2}-\sigma v+\varepsilon}{4 k^{2}\left(\sigma s_{0}-1\right)}$ and $q>0$

$$
Q_{44}(x, t)=e^{i\left(-s_{0} x+w t+\rho\right)}\left[\frac{-q(k+1)(\sigma v-\varepsilon)}{k^{2}\left(\eta s_{0}-\varsigma\right)} \csc _{q}^{2}(x-v t)\right]^{\frac{1}{2 k}} .
$$

where $w=\frac{\varepsilon s_{0}^{2} k^{2}+\alpha s_{0} k^{2}+\sigma v-\varepsilon}{k^{2}\left(\sigma s_{0}-1\right)}$

\subsection{Quadratic-Cubic Law $H(Q)=E_{1} \sqrt{Q}+E_{2} Q$}

From Type 3, we suppose the solution to NPSEs in Equation (34) in the form in Equation (92). The formula in Equation (92) is a solution to Equation (34) when $n=1$, and Equation (34) is integral when $k=1$. Suppose a solution of Equation (34) in the following form:

$$
\psi(\xi)=A_{0}+A_{1} \sec _{q} \xi+B_{1} \tan _{q} \xi .
$$

Equation (113) is a solution of Equation (34) when

Set 1.

$$
A_{0}=-\frac{3(\sigma v-\varepsilon)}{E_{1}}, A_{1}=\frac{3 \sqrt{2 q}(\sigma v-\varepsilon)}{E_{1}}, B_{1}=0, w=\frac{\varepsilon s_{0}^{2}+\alpha s_{0}+2 \sigma v-2 \varepsilon}{\sigma s_{0}-1}, E_{2}=\frac{9 s_{0} \eta(\sigma v-\varepsilon)+E_{1}^{2}}{9(\sigma v-\varepsilon)}, q>0 .
$$

In this set, the solution to NPSEs in Equation (33) is

$$
Q_{45}(x, t)=\left[-\frac{3(\sigma v-\varepsilon)}{E_{1}}+\frac{3 \sqrt{2 q}(\sigma v-\varepsilon)}{E_{1}} \sec _{q}(x-v t)\right] e^{i\left(-s_{0} x+w t+\rho\right)} .
$$

For the type 4. The solutions of the NPSEs in Equation (33) are given by:

$$
Q_{46}(x, t)=\left[-\frac{3(\sigma v-\varepsilon)}{E_{1}}+\frac{3 \sqrt{2 q}(\sigma v-\varepsilon)}{E_{1}} \csc _{q}(x-v t)\right] e^{i\left(-s_{0} x+w t+\rho\right)} .
$$

where $w=\frac{\varepsilon s_{0}^{2}+\alpha s_{0}+2 \sigma v-2 \varepsilon}{\sigma s_{0}-1}$ and $E_{2}=\frac{9 s_{0} \eta(\sigma v-\varepsilon)+E_{1}^{2}}{9(\sigma v-\varepsilon)}, q>0$.

\subsection{Anti-Cubic Law $H(Q)=M_{1} Q^{-2}+M_{2} Q+M_{3} Q^{2}$}

From Type 3, we suppose the solution to NPSEs in Equation (45) in the form in Equation (92). The formula in Equation (92) is a solution to Equation (45) when $n=1$. Suppose a solution of Equation (45) in the following form:

$$
\Omega(\xi)=A_{0}+A_{1} \sec _{q} \xi+B_{1} \tan _{q} \xi
$$

Equation (117) is a solution of Equation (45) when

Set 1.

$$
\begin{gathered}
A_{1}=0, \quad B_{1}=\sqrt{\frac{3(\sigma v-\varepsilon)}{4\left(M_{3}-\eta s_{0}\right)}}, \quad \frac{(\sigma v-\varepsilon)}{\left(M_{3}-\eta s_{0}\right)}>0, w=\frac{4 A_{0}^{2}\left(M_{3}-\eta s_{0}\right)+2 \varepsilon s_{0}^{2}+2 \alpha s_{0}-\varepsilon+\sigma v}{2\left(\sigma s_{0}-1\right)}, \\
M_{2}=-\frac{8}{3} A_{0}\left(M_{3}-\eta s_{0}\right), \quad M_{1}=-\frac{1}{48\left(M_{3}-\eta s_{0}\right)}\left[\left(4 M_{3} A_{0}^{2}+3 \sigma v\right)-\left(3 \varepsilon+4 \eta s_{0} A_{0}^{2}\right)\right]^{2}
\end{gathered}
$$


In this set, the solution to NPSEs in Equation (42) is

$$
Q_{47}(x, t)=\left[A_{0}+\sqrt{\frac{3(\sigma v-\varepsilon)}{4\left(M_{3}-\eta s_{0}\right)}} \tan _{q}(x-v t)\right]^{1 / 2} e^{i\left(-s_{0} x+w t+\rho\right)} .
$$

Set 2.

$$
\begin{gathered}
B_{1}=0, A_{1}=\frac{1}{2} \sqrt{\frac{3 q(\sigma v-\varepsilon)}{\left(M_{3}-\eta s_{0}\right)}}, \quad \frac{q(\sigma v-\varepsilon)}{\left(M_{3}-\eta s_{0}\right)}>0, w=\frac{8 A_{0}^{2}\left(M_{3}-\eta s_{0}\right)+4 \varepsilon \varepsilon_{0}^{2}+4 \alpha s_{0}-\sigma v+\varepsilon}{4\left(\sigma s_{0}-1\right)}, \\
M_{2}=-\frac{8}{3} A_{0}\left(M_{3}-\eta s_{0}\right), \quad M_{1}=-\frac{A_{0}^{2}}{12}\left[4 A_{0}^{2}\left(M_{3}-\eta s_{0}\right)-3(\sigma v-\varepsilon)\right] .
\end{gathered}
$$

In this set, the solution to NPSEs in Equation (42) is

$$
Q_{48}(x, t)=\left[A_{0}+\frac{1}{2} \sqrt{\frac{3 q(\sigma v-\varepsilon)}{\left(M_{3}-\eta s_{0}\right)}} \sec _{q}(x-v t)\right]^{1 / 2} e^{i\left(-s_{0} x+w t+\rho\right)} .
$$

Set 3.

$$
\begin{gathered}
A_{1}=\frac{1}{4} \sqrt{\frac{3 q(\sigma v-\varepsilon)}{\left(M_{3}-\eta s_{0}\right)},}, \quad B_{1}=\frac{1}{4} \sqrt{\frac{3(\sigma v-\varepsilon)}{\left(M_{3}-\eta s_{0}\right)}}, \quad \frac{(\sigma v-\varepsilon)}{\left(M_{3}-\eta s_{0}\right)}>0, q>0, M_{2}=-\frac{8 A_{0}}{3}\left(M_{3}-\eta s_{0}\right), \\
w=\frac{16 A_{0}^{2}\left(M_{3}-\eta s_{0}\right)+8 \varepsilon s_{0}^{2}+8 \alpha s_{0}-\varepsilon+\sigma v}{8\left(\sigma s_{0}-1\right)}, M_{1}=-\frac{1}{768\left(M_{3}-\eta s_{0}\right)}\left[\left(16 M_{3} A_{0}^{2}-3 \varepsilon\right)-\left(16 \eta s_{0} A_{0}^{2}-3 \sigma v\right)\right]^{2}
\end{gathered}
$$

In this set, the solution to NPSEs in Equation (42) is

$$
Q_{49}(x, t)=\left[A_{0}+\frac{1}{4} \sqrt{\frac{3 q(\sigma v-\varepsilon)}{\left(M_{3}-\eta s_{0}\right)}} \sec _{q}(x-v t)+\frac{1}{4} \sqrt{\frac{3(\sigma v-\varepsilon)}{\left(M_{3}-\eta s_{0}\right)}} \tan _{q}(x-v t)\right]^{1 / 2} e^{i\left(-s_{0} x+w t+\rho\right)} .
$$

For the type 4. The solutions of the NPSEs in Equation (42) are given by

$$
Q_{50}(x, t)=\left[A_{0}+\frac{1}{2} \sqrt{\frac{3(\sigma v-\varepsilon)}{\left(M_{3}-\eta s_{0}\right)}} \cot _{q}(x-v t)\right]^{1 / 2} e^{i\left(-s_{0} x+w t+\rho\right)}, \frac{(\sigma v-\varepsilon)}{\left(M_{3}-\eta s_{0}\right)}>0,
$$

where $M_{1}=-\frac{1}{48\left(M_{3}-\eta s_{0}\right)}\left[\left(4 M_{3} A_{0}^{2}+3 \sigma v\right)-\left(3 \varepsilon+4 \eta s_{0} A_{0}^{2}\right)\right]^{2}, M_{2}=-\frac{8}{3} A_{0}\left(M_{3}-\eta s_{0}\right)$ and $w=$ $\frac{4 A_{0}^{2}\left(M_{3}-\eta s_{0}\right)+2 \varepsilon s_{0}^{2}+2 \alpha s_{0}-\varepsilon+\sigma v}{2\left(\sigma s_{0}-1\right)}$

$$
Q_{51}(x, t)=\left[A_{0}+\frac{1}{2} \sqrt{\frac{3 q(\sigma v-\varepsilon)}{\left(M_{3}-\eta s_{0}\right)}} \csc _{q}(x-v t)\right]^{1 / 2} e^{i\left(-s_{0} x+w t+\rho\right)}, \frac{q(\sigma v-\varepsilon)}{\left(M_{3}-\eta s_{0}\right)}>0,
$$

where $M_{2}=-\frac{8}{3} A_{0}\left(M_{3}-\eta s_{0}\right), w=\frac{8 A_{0}^{2}\left(M_{3}-\eta s_{0}\right)+4 \varepsilon s_{0}^{2}+4 \alpha s_{0}-\sigma v+\varepsilon}{4\left(\sigma s_{0}-1\right)}$, and $M_{1}=-\frac{A_{0}^{2}}{12}\left[4 A_{0}^{2}\left(M_{3}-\eta s_{0}\right)-3(\sigma v-\right.$ $\varepsilon)]$.

$$
Q_{52}(x, t)=\left[A_{0}+\frac{1}{4} \sqrt{\frac{3 q(\sigma v-\varepsilon)}{\left(M_{3}-\eta s_{0}\right)}} \csc _{q}(x-v t)+\frac{1}{4} \sqrt{\frac{3(\sigma v-\varepsilon)}{\left(M_{3}-\eta s_{0}\right)}} \cot _{q}(x-v t)\right]^{1 / 2} e^{i\left(-s_{0} x+w t+\rho\right)} .
$$

where $M_{1}=-\frac{1}{768\left(M_{3}-\eta s_{0}\right)}\left[\left(16 M_{3} A_{0}^{2}-3 \varepsilon\right)-\left(16 \eta s_{0} A_{0}^{2}-3 \sigma v\right)\right]^{2}$ and $w=\frac{16 A_{0}^{2}\left(M_{3}-\eta s_{0}\right)+8 \varepsilon s_{0}^{2}+8 \alpha s_{0}-\varepsilon+\sigma v}{8\left(\sigma s_{0}-1\right)}$, $q>0$ 


\section{Behavior of Soliton Solutions}

In this section we plot the two- and one-dimensional solutions when the parameters take some suitable values to determine the type of the optical solutions. We show the effect of the parameter $q$ in the q-deformed functions in optical solutions. We plot the absolute value of the complex exact solution to discuss the amplitude of the soliton solutions. In Figure 1, we plot the traveling wave solution (17) when the parameters take the values $s_{0}=0.01, \varepsilon=2, \alpha=5, \sigma=0.5, \eta=1, \zeta=-100, A=3$ and $B=2$. We get a singularity at $x=-1.4$ when the velocity of solion is equal to 2 and the wave number is 4; consequently the soliton solution in Equation (17) is the singular soliton solution while in the Figure 2, the soliton solutions in Equation (18) is periodic singular solution. Figure 3 presents the exact solution of Equation (59) when $s_{0}=2, \varepsilon=0.2, \alpha=-2, \sigma=0.1, \eta=2, \zeta=1, q=11$ is the bright soliton equations while the exact Equation (80) in the Figure 4 is dark soliton equations. Figure 5 illustrates the periodic soliton Equation (119) when $s_{0}=2, \varepsilon=0.2, \alpha=-2, \sigma=0.1, \eta=2, \zeta=1, q=11$.

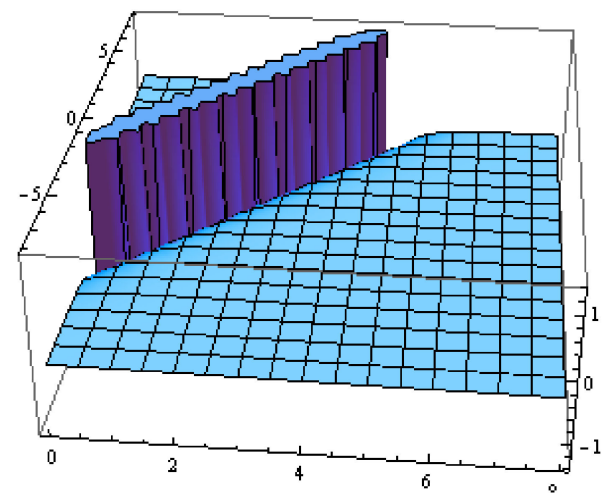

(a)

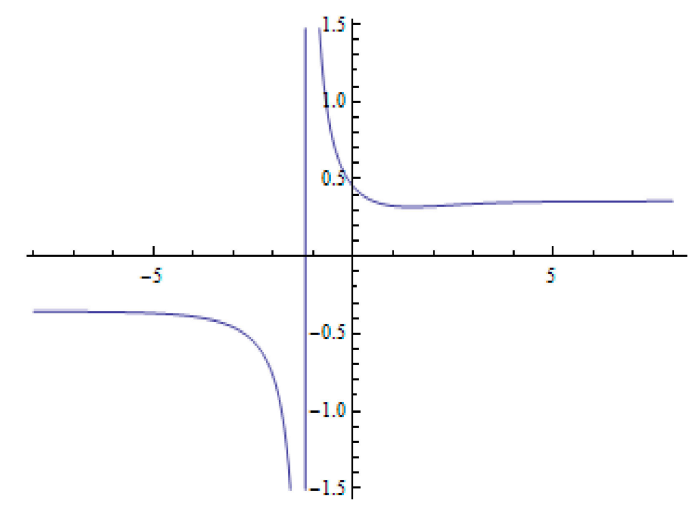

(b)

Figure 1. (a) The Absolute value of exact solution $Q_{1}$ when $s_{0}=2, \varepsilon=0.2, \alpha=-2, \sigma=0.1, \eta=2, \zeta=$ $1, A=3$ and $B=2$ and $(\mathbf{b})$ is the projection $Q_{1}$ in $2 \mathrm{D}$.

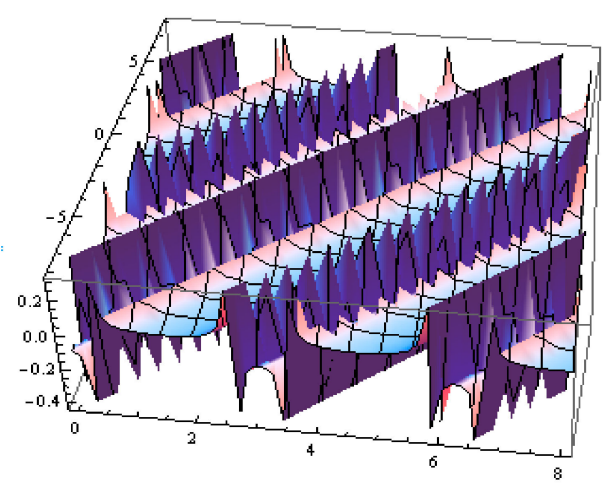

(a)

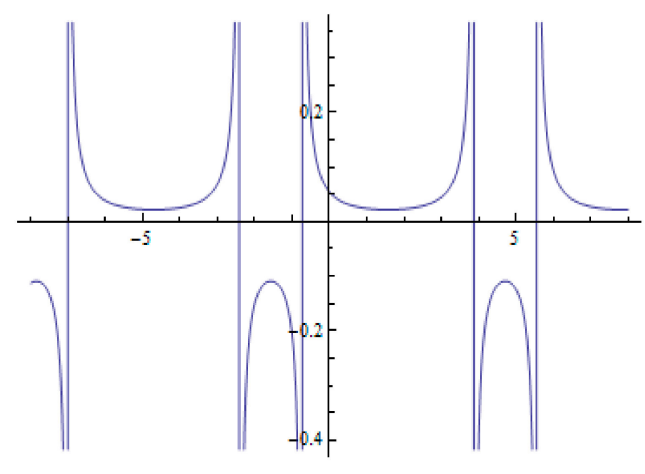

(b)

Figure 2. (a) The Absolute value of exact solution $Q_{2}$ when $s_{0}=2, \varepsilon=0.2, \alpha=-2, \sigma=0.1, \eta=2, \zeta=$ $1, A=3$ and $B=2$ and $(\mathbf{b})$ is the projection $Q_{2}$ in $2 \mathrm{D}$. 


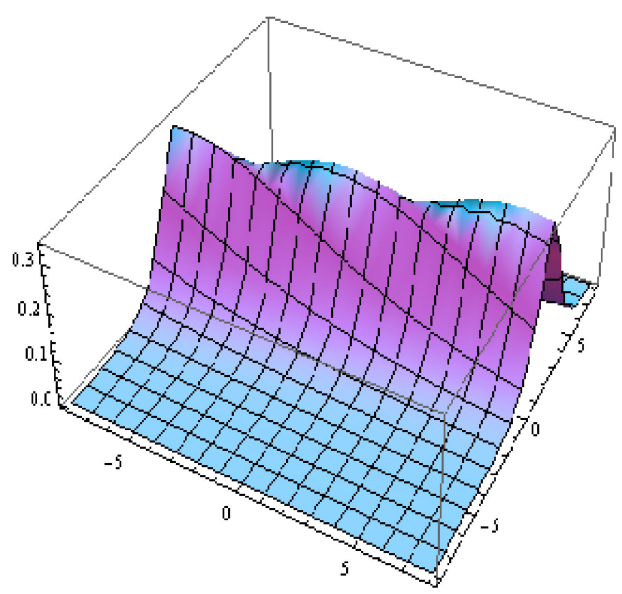

(a)

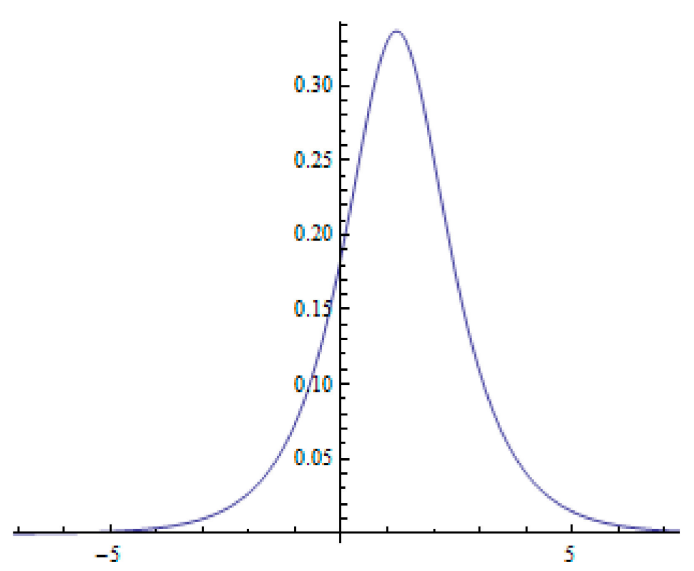

(b)

Figure 3. (a) The Absolute value of exact solution $Q_{17}$ when $s_{0}=2, \varepsilon=0.2, \alpha=-2, \sigma=0.1, \eta=2, \zeta=$ $1, q=11$ and (b) is the projection $Q_{17}$ in $2 \mathrm{D}$.

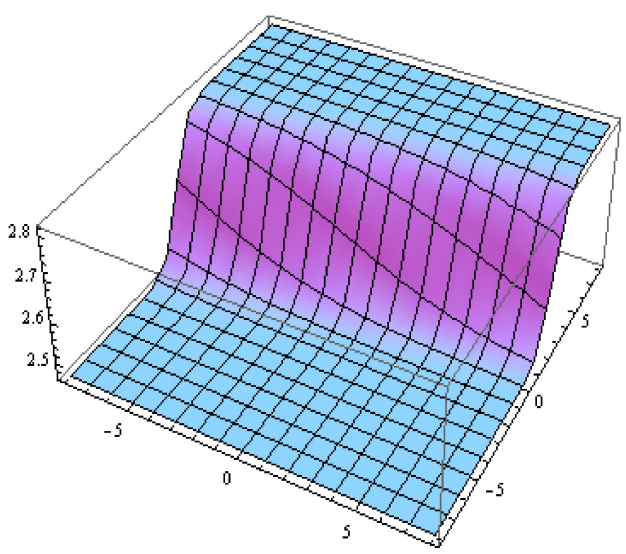

(a)

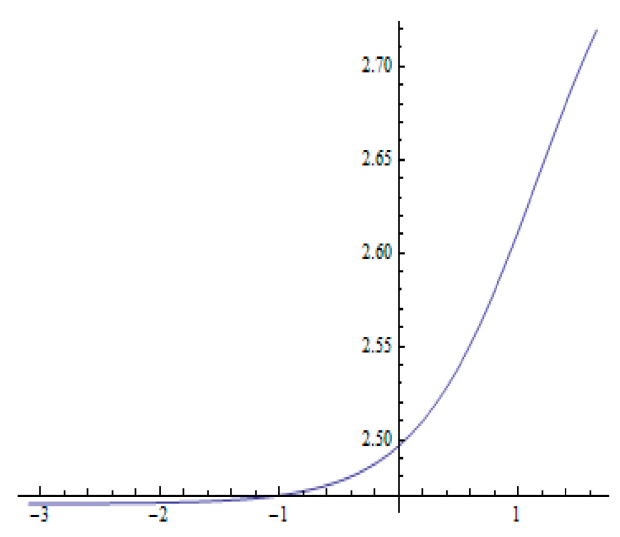

(b)

Figure 4. (a) The Absolute value of exact solution $Q_{28}$ when $s_{0}=2, \varepsilon=0.2, \alpha=-2, \sigma=0.1, \eta=2, \zeta=$ $1, q=11$ and (b) is the projection of $Q_{28}$ in $2 \mathrm{D}$.

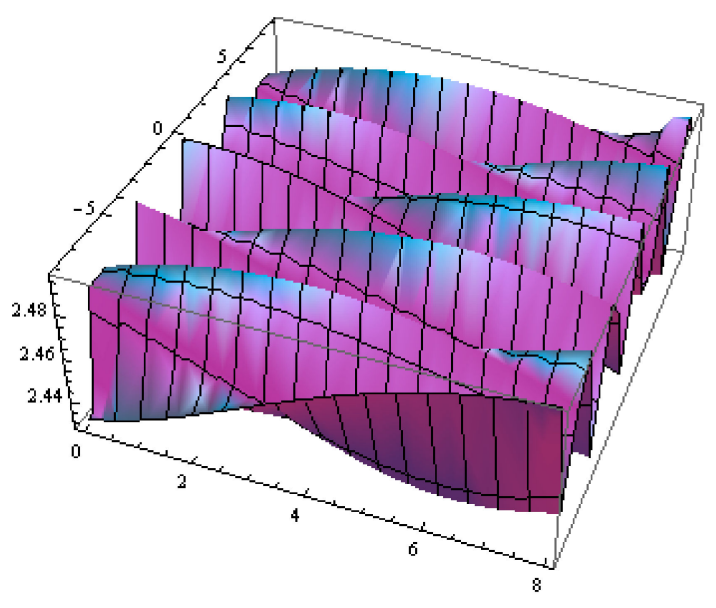

(a)

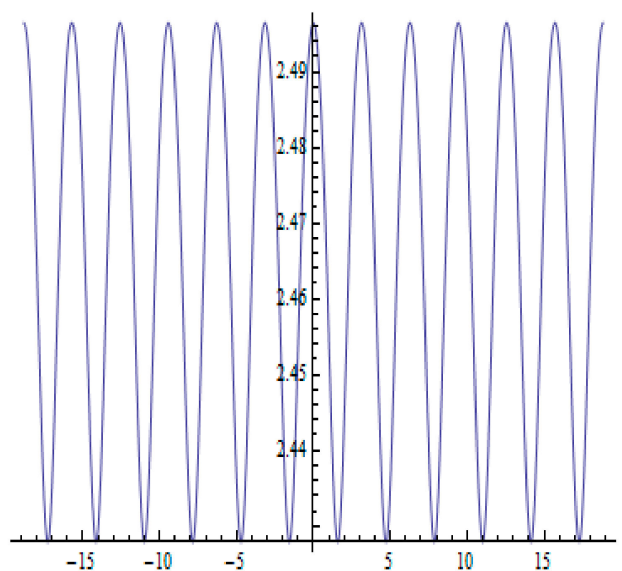

(b)

Figure 5. (a) The Absolute value of exact solution $Q_{47}$ when $s_{0}=2, \varepsilon=0.2, \alpha=-2, \sigma=0.1, \eta=2, \zeta=$ $1, q=11$ and (b) is the projection of $Q_{47}$ in $2 \mathrm{D}$. 


\section{Conclusions}

In this paper, we applied some interesting algebraic methods to find a variety of explicit solutions to nonlinear perturbed Schrödinger equations when the nonlinear parts take different terms: nonlinear Kerr law, power law, quadratic-cubic law, and anti-cubic law. In this article, we construct the rational solitary solutions, q-deformed hyperbolic solutions, and q-deformed trigonometric solutions for one of the important equations in optical media which has many applications in optics and communications. Through our study that we conducted on the non-linear Schrödinger equation, it is clear that the proposed methods are effective methods in finding the soliton solutions. The drawings studied during this work illustrate the different optical solutions that have many applications in optics.

Funding: Taif University Researchers Supporting project number (TURSP-2020/16), Taif university, Taif, Saudi Arabia.

Acknowledgments: The author thanks Taif university researchers for supporting project number (TURSP-2020/16), Taif university, Taif, Saudi Arabia.

Conflicts of Interest: The authors declare no conflict of interest.

\section{References}

1. Biswas, A.; Konar, S. Introduction to Non-Kerr Law Solitions; CRC Press: Boca Raton, FL, USA, 2005.

2. Arshed, S.; Arif, A. Soliton solutions of higher-order nonlinear Schrödinger equation and nonlinear Kudryashov's equation. Opt. Int. J. Light Electron. Opt. 2020, 209, 164588. [CrossRef]

3. Arshed, S. New soliton solutions to the perturbed nonlinear Schrodinger equation by exp $(-\phi(x))$-expansion method. Opt. Int. J. Light Electron. Opt. 2020, 220, 165123. [CrossRef]

4. Wazwaz, A.M. A variety of optical solitons for nonlinear schrödinger equa- tion with detuning term by the variational iteration method. Optik 2019, 196, 163169. [CrossRef]

5. Zayed, E.E.; Abourabia, A.M.; Gepreel, K.A.; Horbaty, M.E. On the rational solitary wave solutions for the nonlinear Hirota-Satsuma coupled KdV system. Appl. Anal. 2006, 85, 751-768. [CrossRef]

6. Gepreel, K.A.; Mahdy, A.M.S.; Mohamed, M.S.; Al-Amiri, A. Reduced Differential Transform Method for Solving Nonlinear Biomathematics Models. Comput. Mater. Contin. 2019, 61, 979-994. [CrossRef]

7. Deta, U.A. Suparmi. The properties of Q-deformed hyperbolic and trigonometric functions in quantum deformation. In AIP Conference Proceedings; AIP Publishing LLC: Melville, NY, USA, 2015; Volume 1677, p. 4930629.

8. Ahmed, N.; Irshad, A.; Mohyud-Din, S.T.; Khan, U. Khan, Exact solutions of perturbed nonlinear Schrödinger'sequation with Kerr law nonlinearity by improved $\tan \left(\frac{\varphi}{2}\right)$ expansion method. Opt. Quant. Electron. 2018, 50, 45. [CrossRef]

9. Gao, H.; Xu, T.; Wang, G. Optical Solitons for the Perturbed Nonlinear Schrödinger Equation with Kerr Law and Non-Kerr Law Nonlinearity. Z. Nat. A 2018, 73, 315-321. [CrossRef]

10. Rizvi, S.T.; Ahmad, S.; Nadeem, M.F.; Awais, M. Optical dromions for perturbed nonlinear Schrödinger equation with cubic quintic septic media. Opt. Int. J. Light Electron. Opt. 2020, 226, 165955. [CrossRef]

11. Savaissou, N.; Gambo, B.; Rezazadeh, H.; Bekir, A.; Doka, S.Y. Exact optical solitons to the perturbed nonlinear Schrödinger equation with dual-power law of nonlinearity. Opt. Quantum Electron. 2020, $52,192$. [CrossRef]

12. Marinkovi, S.; Stankovi, M.; Mulali, E. The deformed trigonometric functions of two variables. Math. Balk. New Ser. 2012, 26, 147-158.

13. Li, J.; Han, M. Exact peakon solutions given by the generalized hyperbolic functions for some nonlinear wave equations. J. Appl. Anal. Comput. 2020, 10, 1709-1719.

14. Houwe, A.; Abbagari, S.; Betchewe, G.; Inc, M.; Doka, S.Y.; Crépin, K.T.; Baleanu, D.; Almohsen, B. Exact optical solitons of the perturbed nonlinear Schrödinger-Hirota equation with Kerr law nonlinearity in nonlinear fiber optics. Open Phys. 2020, 18, 526-534. [CrossRef]

15. Ghanbari, B.; Raza, N. An analytical method for soliton solutions of perturbed Schrödinger's equation with quadratic-cubic nonlinearity. Mod. Phys. Lett. B 2019, 33, 1950018. [CrossRef] 
16. Khater, M.; Baleanu, D.; Abdel-Aty, A.; Khalil, E.; Bouslimi, J.; Omri, M. Abundant distinct types of solutions for the nervous biological fractional FitzHugh-Nagumo equation via three different sorts of schemes. Adv. Differ. Equ. 2020, 2020, 476.

17. Gepreel, K.A.; Nofal, T.A.; Al-Asmari, A.A. Traveling wave solutions for Nonlinear Kawahara Partial Differential Equation Using Extended Trial Equation Method. Int. J. Comput. Math. 2019, 96, 1357-1376. [CrossRef]

18. Stegeman, G.I.; Segev, M. Optical Spatial Solitons and Their Interactions: Universality and diversity. Science 1999, 286, 1518-1523. [CrossRef]

19. Segev, M.; Crosignani, B.; Yariv, A. Spatial solitons in photorefractive media. Phys. Rev. Lett. 1992, 68, 923-927.

20. Chowdury, A.; Krolikowski, W.; Akhmediev, N. Breather solutions of a fourth-order nonlinear Schrödinger equation in the degenerate, soliton, and rogue wave limits. Phys. Rev. Lett. 2017, 96, 042209.

21. Dobrogowska, A. The q-deformation of Hyperbolic and Trigonometric Potentials. Int. J. Differ. Equ. 2014, 9, 45-51.

22. Eğrifes, H.; Demirhan, D.; Büyükkılıç, F. Exact solutions of the Schrodinger equation for the deformed hyperbolic potential well and the deformed four-parameter exponential type potential. Phys. Lett. A 2000, 275, 229-237. [CrossRef]

23. Ma, W.X.; Chen, M. Direct search for exact solutions to the nonlinear Schrodinger equation. Appl. Math. Comput. 2009, 215, 2835-2842. [CrossRef]

24. Ma, W.X.; Zhang, L. Lump solutions with higher-order rational dispersion relations. Pramana 2020, 94, 43. [CrossRef]

25. Ma, W.X. Lump and interaction solutions to linear PDEs in $2+1$ dimensions via symbolic computation. Mod. Phys. Lett. B 2019, 33, 1950457. [CrossRef]

26. Ma, W.X.; Lee, J. A transformed rational function method and exact solutions to the 3+1 dimensional Jimbo-Miwa equation. Chaos Solitons Fractals 2009, 42, 1356-1363. [CrossRef]

Publisher's Note: MDPI stays neutral with regard to jurisdictional claims in published maps and institutional affiliations.

(C) 2020 by the author. Licensee MDPI, Basel, Switzerland. This article is an open access article distributed under the terms and conditions of the Creative Commons Attribution (CC BY) license (http://creativecommons.org/licenses/by/4.0/). 